\title{
A Compact Modular Soft Surface with Reconfigurable Shape and Stiffness
}

\author{
Matthew A. Robertson, Student Member, IEEE, Masato Murakami, Wyatt Felt, Member, IEEE \\ and Jamie Paik, Member, IEEE
}

\begin{abstract}
A variety of reconfigurable surface devices, utilizing large numbers of actuated physical pixels to produce discretized 3D contours, have been developed for different purposes in research and industry. The difficulty of integrating many actuators in close configuration has limited the DoF and resolution and performance of existing devices. Utilizing vacuum power and soft material actuators, we have developed a soft reconfigurable surface (SRS) with multi-modal control and performance capabilities. The SRS is comprised of a square grid array of linear vacuum-powered soft pneumatic actuators (linear V-SPAs), built into plug-and-play modules which enable the arrangement, consolidation, and control of many DoF. In addition to the practical benefits of system integration, this architecture facilitates the construction of customized assemblies with an overall compact form factor. A series of experiments is performed to illustrate and validate the versatility of the SRS for achieving diverse tasks including force controlled modulation of interface pressure through integrated sensors, lateral manipulation of a variety of objects, static and dynamic shape and pattern generation for haptic interaction, and variable surface stiffness tuning. This SRS concept is scalable, space efficient and features diverse functional potential. This will extend the utility and accessibility of tangible robotic interfaces for future applications from industrial to home and personal use.
\end{abstract}

Index Terms-Soft Robotics, Pneumatics, Vacuum, Peristaltic Table, Reconfigurable Surface, Haptic Interface.

\section{INTRODUCTION}

A reconfigurable surface is a nominally 2D structure which can be transformed to render 3D shapes and contours. This is often achieved by discretizing the surface into an array of height-variable physical pixels, pins or rods which are used to construct digitized or physically interpolated (via a flexible covering) geometric features [1], [2]. This concept has attracted interest for diverse applications in a variety of interactive and physical user interfaces.

In the field of Human-Computer Interaction, reconfigurable surfaces are considered as a subclass of "shape-changing interfaces" [3]. In this field, reconfigurable surfaces may be used, for example, to create physically $3 \mathrm{D}$ visual displays [4][7]. The ideas behind these displays have found their way into (and likely out of) science fiction movies such as X-Men (2000) [8].

The changes in surface elevation can also be used to create tactile displays for the visually impaired. Such is the goal of "refreshable braille displays" [9]. These devices display different braille characters by toggling pins between raised

Reconfigurable Robotics Laboratory (RRL), Department of Mechanical Engineering, École Polytechnique Fédérale de Lausanne (EPFL), Lausanne, Switzerland. Correspondance: jamie.paik@epfl.ch and lowered positions. Though traditionally these devices have relied on relatively large piezoelectric actuators, proposed actuation concepts include microfluidics [10]-[12], electroactive polymers [13]-[15] and shape-memory alloys [16]-[18].

Other displays have been designed to render tactile or haptic information to unimpaired users. For example, inflating pneumatic cells have been combined with a jamming-particle surface to create a haptic surface that can render different shapes and mechanical stiffness [19], [20]. Another display, FEELEX, includes force sensors on each pixel, allowing it to react to the interaction forces from the user [7]. These tactile displays can also be placed on the body to provide tactile cues without visual distraction [21]-[24].

The principles behind these shape/pressure adjusting technologies are also found in more personal applications. For instance, medical mattresses can be overlaid with "dynamic support surfaces" that actively alternate or adjust pressure distributions to prevent bedsores [25]. Air bladders or mechanical devices allow the seats of luxury automobiles to adapt to different users and preferences [26]. And the ubiquitous "massage chair" uses its surface to create dynamic pressures on the back, legs or feet of its users.

A changing topology can do more than just change its shape or pressure profile, it can also transport objects on its surface. This may be accomplished, for instance, by creating wave-like profiles that cause the object to roll or slide in the desired direction [4], [27], [28]. FESTO's "WaveHandling" system relies on a network of bellows under a flexible surface [27]. A braille-display-controlled manifold of soft blisters has been used to control the rolling of a ball [28]. Other surfaces can move flat objects without rolling. Taking inspiration from the locomotion of caterpillars, these "soft tables" can manipulate objects in three degrees of freedom by controlling the elevation and translation of an array of soft legs [29].

In the manufacturing industry, controllable topology has been leveraged to develop reconfigurable die and mold tooling concepts for reducing production costs. This method allows forming of sheet materials with different curvature patterns or shapes using a single mold. This technique has been proposed for sheet metal forming [30], [31] and composite molding [32]. A commercially available "adaptive mould", produced by Adapa, can aid the production of custom double-curved panels out of composites, thermoplastics, concrete, and glass [33].

Every reconfigurable surface device with many DoF involves a significant challenge in the integration and control of a large number of actuators necessary for its function. Differ- 
ent designs are constrained by different practical limitations. Purely mechanical systems require either very small actuators embedded below each physical pixel, or transmission elements such as flexible cables to relay force from actuators separated from the active surface elements by distance and scale. Typically the latter results in systems which are much larger than the functional area of the reconfigurable surface itself, which limits the applications of such devices to effectively stationary and unconstrained environments. Other surfaces which are driven by small embedded actuators usually rely on such mechanisms as smart materials including SMAs, electroactive polymers, piezoelectic actuators, or miniaturized voice-coil linear motors [9], [34]. While principally ideal for applications matching their physical scale, these technologies are generally characterized by low force, and stroke limitations. Other solutions which utilize fluid as a transmission medium often combine both the advantages and disadvantages of both types of systems; incorporating compact actuators while relying on inefficient architecture for managing fluid power distribution and control. Some progress has been made in existing work to address these challenges, specifically through the development of modular soft actuators which were leveraged to produce robot morphologies powered by pneumatic vacuum that could not have otherwise been implemented practically [35]. The objective of this work is to study the performance of physical shape and load modulation control modes for tangible interactions and haptic interfacing using a modular, high-DoF, reconfigurable surface powered by pneumatic vacuum through a single port. This contribution will extend the existing utility of reconfigurable surface systems and present new alternative uses or practical potential to leverage further benefits.

We developed a new, compact interactive platform, a soft reconfigurable surface (SRS), based on a modular actuator network to test applications in safe object manipulation, haptic interaction and variable-stiffness surfaces. The SRS concept we present is modular, allowing the "plug and play" modules to be rearranged into arbitrary footprints with little design effort. This modular, embedded hardware approach also facilitates the use of versatile and powerful actuators while maintaining an overall space and weight efficient embodiment. Our system's unique combination of performance potential, physical design efficiency, and customizability could lead to use in new, unexplored domains, including personal and portable use outside of industrial and scientific research settings.

\section{Methods}

\section{A. Physical System Architecture}

1) Base platform: The SRS prototype shown in Fig. 1 is comprised of 16 modular actuator units arranged in a $4 \times 4$ grid layout. This configuration is chosen to enable a diverse variety of system capabilities and behaviors, however due to their modular construction, any arrangement of actuators is possible. A functional base platform dictates the overall size, shape, and resolution of the final system and may be easily customized. The platform provides mechanical interface features which allows actuator modules to be plugged into it
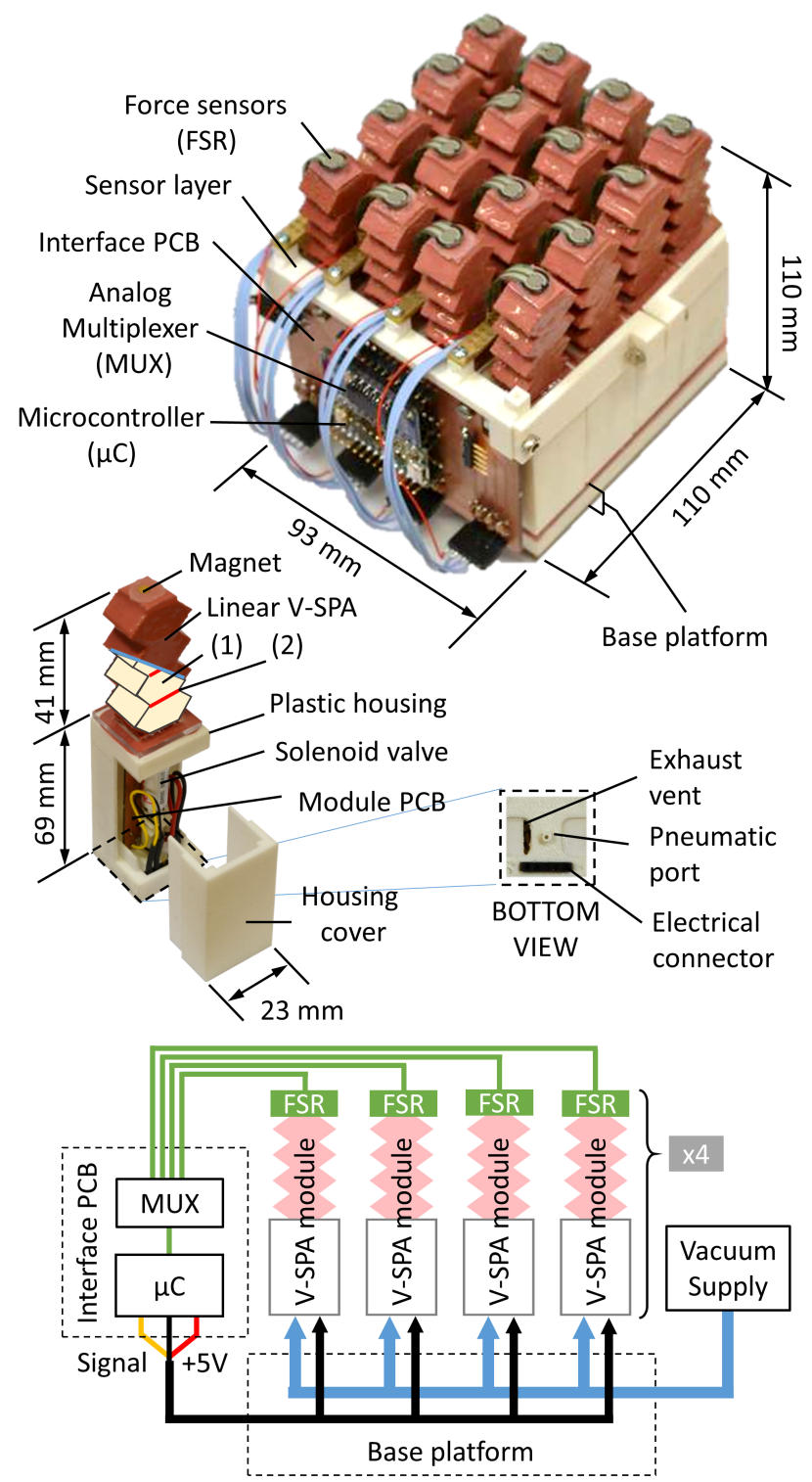

Fig. 1. The complete SRS prototype is shown, comprised of 16 independently controllable single DoF modular actuators powered by vacuum pneumatic pressure, a force sensor layer, and control and interface electronics. Each actuator module is interconnected through a multi-port interface from below to a base platform which provides pneumatic and electrical power supply in addition to a computer control signal. A module consists of a single control valve, communication electronics, and a Linear V-SPA, shown with an illustrated cross section depicting the foam chambers (1), and dividing membranes (2).

and physically joined to form a single system. The base also serves as a pneumatic supply manifold and electrical control network interconnection. For fluid power, every module is connected through a port to a shared negative pressure supply distributed through parallel branched channels embedded in the base manifold. Only a single pneumatic supply line is required to power the base platform, irrespective of the its shape, or the number of modules attached to it. For electrical power, the modules are also connected in parallel, while for control each module is electrically connected through the base platform in series on an extensible single wire communication 
bus.

2) Module: The module body contains embedded hardware and electronics which enable attached actuators to be powered and controlled locally. The integrated components include one solenoid valve (Lee, LHDA0531115H) mounted internally on a Printed Circuit Board (PCB) and a communication IC (Worldsemi, WS2811) that allows commands to be sent to every module independently over a single signal line. These decentralized components are key to the practicality of the final system as they reduce the complexity of connecting many pneumatic actuators required to achieve high-DoF in the reconfigurable surface. The modular architecture also yields a complete system which is relatively compact compared to other reconfigurable surface systems.

3) Linear V-SPA: A linear vacuum-powered soft pneumatic actuator (V-SPA) is attached to the top of the module body. When combined with other modules in close arrangement, the distal endpoints of the actuators constitute an interactive, variable geometry surface. To render adaptable shapes or stiffness profiles, the vertical height of each actuator is controlled by modulation of a vacuum pressure pneumatic supply. In this way every actuator module only has a single DoF while the system as a whole consequently consists of 16 independent DoF.

Linear V-SPAs are composed of an open-cell polyurethane foam core assembled in separate pieces sequentially between stiff paper dividers, separating the whole actuator into a stack of multiple chambers following methods and materials similar to previous work [35]. To accommodate the attachment of sensors to the end of the actuator, a rigid $1 \mathrm{~mm}$ thick acrylic plate is glued to the top of the core. The linear core assembly is then manually coated with two thin layers of Elastosil M4606 silicone rubber to seal it from the environment, leaving an uncoated portion at the proximal end to attach to a mounting plate with a hole for the pneumatic supply. Under the negative pressure of the vacuum supply, the actuator is driven to collapse. The relatively stiff paper dividers and the hexagonal shape of each foam core segment directs the structure to create large compressions in the actuator length. With the actuators oriented vertically at the top of the modular units, this retraction produces a height displacement which changes the shape and contour approximated by the endpoints of all the actuator modules combined in the SRS. By controlling the vacuum pressure in the modules we can modulate the actuator position, end-force, or effective stiffness.

4) Sensors: To enable optional feedback control of the SRS, an additional modular force-sensing layer can be attached to the assembled grid of actuator modules. The sensor layer is comprised of a $3 \mathrm{D}$ printed $\mathrm{ABS}$ plastic structure which surrounds and clamps onto the assembly, and houses thin PCB rails which fit between the rows of linear V-SPAs comprising the upper portion of the SRS. Adjacent to every V-SPA along the rails, the thin, flat cable of a $4 \mathrm{~mm}$ diameter flexible Force Sensitive Resistor (FSR 400, Interlink Electronics) is attached. The actuator modules as well as the sensor layer are connected through an interface PCB which houses a microcontroller unit (Teensy LC) and an analog multiplexer which facilitates reading all 16 surface sensors from a single analog and four digital microcontroller inputs. The PCB also contains a threewire control connection header to attach the actuator to the module base platform, and a final header to receive highcurrent $5 \mathrm{~V}$ power from an external supply (every valve draws $150 \mathrm{~mA}$ current, for a total of $2.4 \mathrm{~A}$ possible when all modules are active).

To physically interface with the actuators, a small, highforce neodymium magnet is glued to the back side of the functional, distal end of the sensor, which is oriented to compliment a magnet of opposing polarity embedded in the end of the surface actuator. This allows removable temporary attachment or detachment of the sensor assembly to the main SRS body. When the actuator modules and sensor layer are combined, each actuator is paired through software to the sensor attached to its end, enabling individual force control of every actuator in the complete surface.

\section{B. System control}

1) Experimental Module Quasi-static Characterization: A single actuator module was tested in compression with vacuum gauge pressure levels between $0 \mathrm{kPa}$ and $-30 \mathrm{kPa}$. These tests were conducted under quasi-static conditions in an Instron 5965 with a $500 \mathrm{~N}$ load cell. A tack was glued to the top of the module to keep it oriented to the load-cell during compression. The long-slender actuator buckled and rebounded under the compressive loads. A polynomial surface was fit to the data. This surface related the compression (5th-order regression) and pressure levels (2nd-order regression) to the measured load. The results of this regression are shown in Fig. 2a. The $R^{2}$ value of the regression was 0.933 and the RMS of the residual error was $0.427 \mathrm{~N}$. The smooth polynomial function used for the regression allowed was used in the analysis of the module behavior.

2) Position Control: The polynomial surface regressed to the module characterization data can be used to identify an approximate relationship between actuator free contraction and pressure (Fig. 2b). The true behavior of the actuator for a given pressure will vary from the curve shown in Fig. $2 b$ because of hysteresis. The maximum free contraction, with $-30 \mathrm{kPa}$, was approximately $1 \mathrm{~cm}$ (for a nominal relaxed module height of $4 \mathrm{~cm})$.

3) Force Control: The force at the actuator tip comes from the compression of the foam within the actuator (Fig. 3a). For small levels of compression, only small forces can be achieved. For moderate levels of compression, the force remains roughly constant. For example, between $5 \mathrm{~mm}$ and $12 \mathrm{~mm}$ of contraction, the maximum tip force as characterized by the polynomial regression increases from $1.8 \mathrm{~N}$ to only $2.3 \mathrm{~N}$. This force can be modulated lower through the application of vacuum pressure. For compressions smaller than $1 \mathrm{~cm}$, the output force can be decreased to zero (with a maximum vacuum gauge pressure of $-30 \mathrm{kPa}$. Beyond this point, the force can still be decreased but the change in force, $\Delta F$, achievable through vacuum decreases gradually until $2 \mathrm{~cm}$ of compression is reached.

4) Stiffness Control: The vacuum pressure can be used to modulate the passive stiffness of the modules. For a module at 

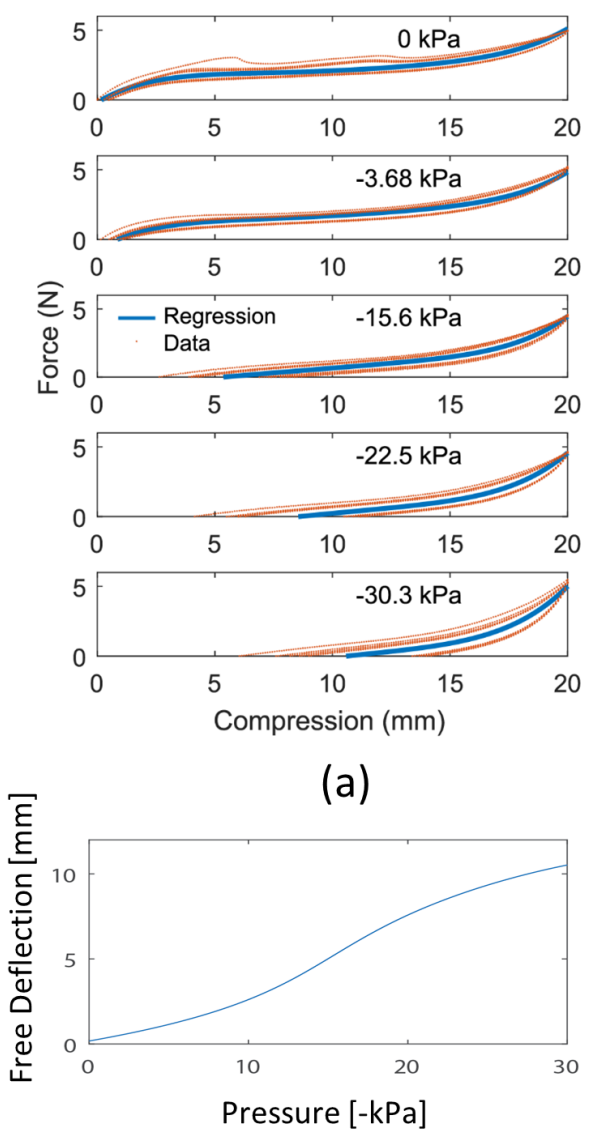

(b)

Fig. 2. (a) An individual actuator module was tested in compression Increasing the magnitude of the vacuum pressure increases the free deflection of the actuator. To compress the actuator beyond this point, external forces are required. This load-behavior of the actuator was captured well by a twovariable (compression and pressure) polynomial surface. (b) The relationship shown here between the point of zero external force and pressure was identified from the polynomial surface regressed to the characterization data.

a given free displacement, the stiffness quantifies how quickly the force increases for a given displacement beyond its unforced level. The partial derivative (with respect to displacement) of the polynomial surface for module force can provide a measure of stiffness. Evaluating this partial derivative at the free-displacement point (Fig. 3b) shows that increasing the magnitude of the vacuum pressure decreases the stiffness of the system for small deflections. This small-deflection stiffness decreases from $0.94 \mathrm{~N} / \mathrm{mm}$ with no pressure to $0.17 \mathrm{~N} / \mathrm{mm}$ at $30 \mathrm{kPa}$, an $82 \%$ reduction. At larger displacements (e.g. $1 \mathrm{~cm})$, the instantaneous stiffness increases for pressure values with magnitudes above about $-15 \mathrm{kPa}$. At this deflection beyond the no-force point, the instantaneous stiffness can be as high as $1.9 \mathrm{~N} / \mathrm{mm}$.

\section{RESULTS}

We validate the practical capability of the SRS for use in different applications by demonstrating a variety of distinct, programmable control modes. Utilizing integrated force sensors coupled to every linear V-SPA module, we present

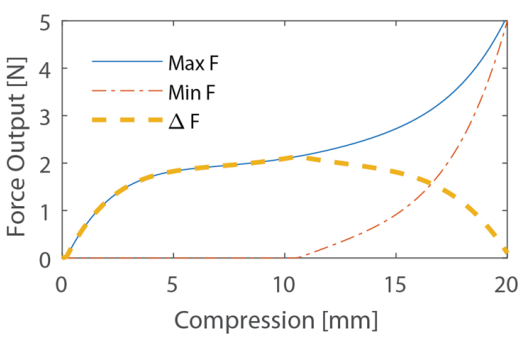

(a)

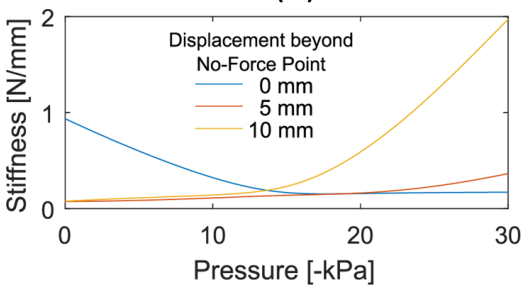

(b)

Fig. 3. (a) The achievable force output of the actuator is constrained by its compression level. At low-levels of compression, only small forces can be achieved. The largest range of achievable forces occurs at approximately $1 \mathrm{~cm}$ of compression. As the compression approaches $2 \mathrm{~cm}$, higher forces can be achieved but the vacuum pressure loses its ability to reduce the force from the foam. (b) The instantaneous stiffness of the actuator (evaluated at the free deflection point) decreases with increasing magnitudes of vacuum pressure. An approximately five-fold decrease in stiffness (over small deflections) can be achieved. With vacuum pressure magnitudes above about $-15 \mathrm{kPa}$ and for compressions on the order of $1 \mathrm{~cm}$ beyond the no-force point, the effective stiffness of the modules increases with increasing magnitudes of vacuum pressure.

the operation of the SRS in force control mode for interface pressure redistribution and input device functionality. An alternate mode, controlling actuator position in open-loop and neglecting the stiffness change coupled to displacement, is shown to generate different shapes and patterns for haptic display or direct physical manipulation of external objects. Finally, leveraging the variable stiffness characteristic of the actuators as a function of pressure, we achieve a stiffness control mode which yields a soft surface with tunable passive compliance.

\section{A. Force Mode: Interface Pressure Modulation}

Closed-loop force control was implemented for each linear V-SPA module to permit active modulation of the interface pressure (IP) between the top of the SRS and an external load. Individual sensors were attached to the endpoint of each linear V-SPA and a binary (bang-bang) control scheme was employed to achieve two different force modulation capabilities: IP redistribution and IP reduction.

The same simple control strategy resulted in the behavior for both types of loading. Using the control with a fixed load results in an IP redistribution behavior. Fixed displacements result in an overall IP reduction. These scenarios are analogous to different potential applications for a compact SRS, including an active pressure-point reductive bed or seating surface (fixed load) or dynamically adjustable body interface for wearable robotic devices (fixed displacement). Fig. 4 illustrates these behaviors. 


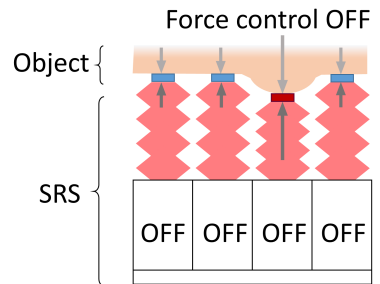

( $r 3$ side view)

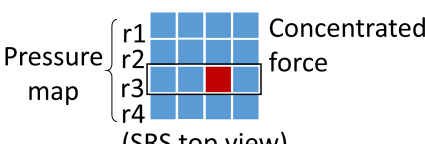

(SRS top view)

Fixed force

Fixed displacement

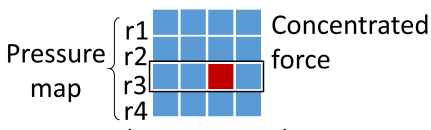

(SRS top view)

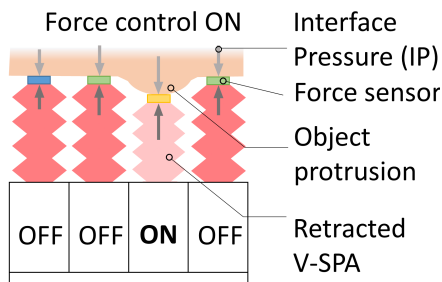

( $r 3$ side view)

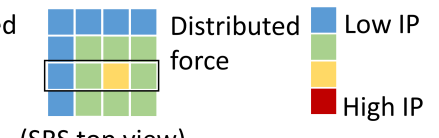

(SRS top view)

Load redistribution Load reduction

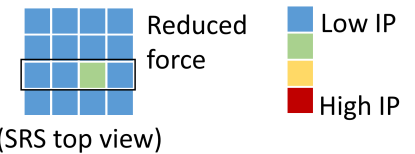

Fig. 4. Through retraction of the actuators we compensate for uneven interface pressure distributions based on contact force detection. For a fixed force load, representing an object resting on the surface, interface pressure concentrated at a single point is distributed among other actuators when the load is reduced at the concentration point. For fixed displacement loads, the reduction of interface pressure (IP) at the concentration does not affect adjacent areas of the surface.

The performance of the force control mode was evaluated through a set of tests designed to simulate these potential use conditions and illustrate the efficacy of the different control behaviors. Fig. 5 shows the results from the validation experiments performed using the SRS and various physical test objects used to produce variable load distribution. For both test conditions, two force targets were tested, corresponding to $29 \%$ and $68 \%$ of the maximum measurable force for a single sensor (roughly $1 / 3$ and $2 / 3$ of the sensor range respectively, to demonstrate two distinct targets within the nominal regime of the sensor separated from both extremes at the minimum and maximum of the range).

For the fixed load tests, a square, rigid medium-density fiberboard (MDF) plate was placed resting on top of the surface, to support an object of $739 \mathrm{~g}$ mass. In addition to the plate with a flat contour on both sides, three other interface plates were tested with protrusions around the edge, in the center, and in the corner. These plate variations produced different interface pressure patterns used to highlight the effect of the active force control. A measurement was initially taken of all 16 sensors with the force control deactivated, and then again after it was turned on.

For the fixed displacement tests, a heavy duty scientific ring stand was used hold the MDF plate against the surface at a pre-set deflection of $3 \mathrm{~mm}$ below the nominal, unpowered resting height of the actuators. Again, measurements were made before and after activation of the force control.

\section{B. Position Mode: Shape and Pattern Generation}

1) Tactile display: The SRS can be utilized as an output device to physically display meaningful information through
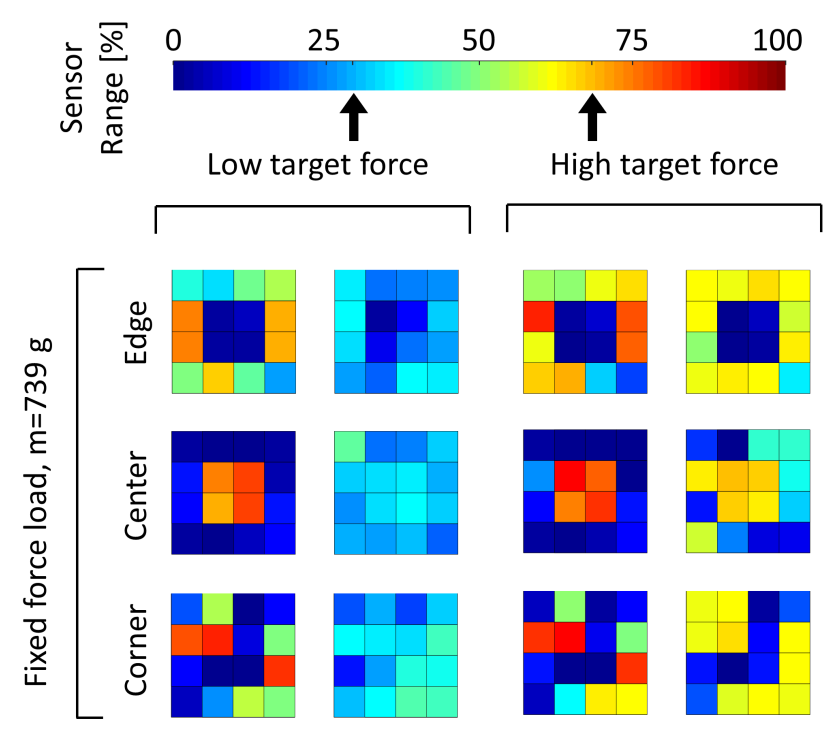

(a)

(b)
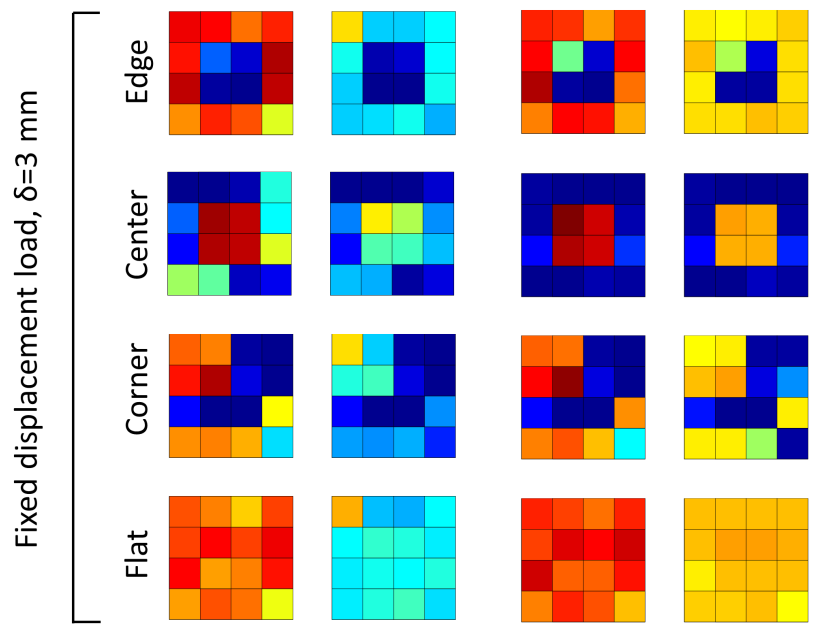

(c)
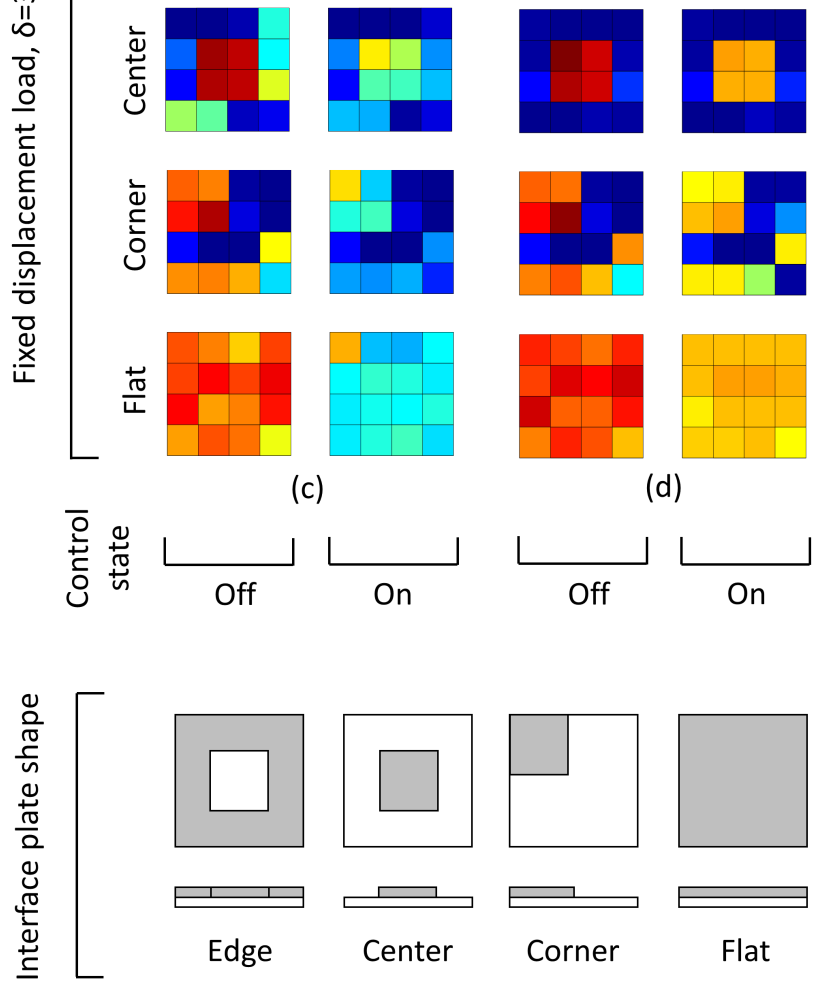

Fig. 5. Experimental results using closed-loop control to modulate the surface IP. Different patterns of non-uniform IP were imposed by interface plates in contact with the surface, with a variety of physical profiles. The physical profiles on the interface plates included protrusions at the edge, center, or corner of the surface. The pressure maps between controller off and on states show the effect of the active force control. With fixed loads, the IP was redistributed and with fixed displacements, it was reduced. 
(a)
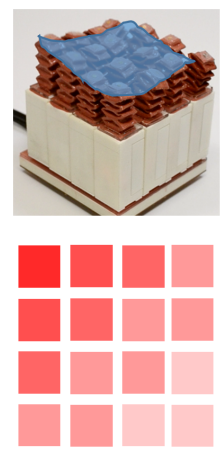

3D Topo

(b)

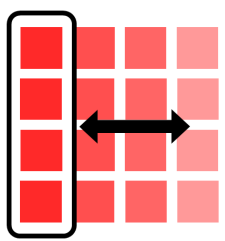

(c)

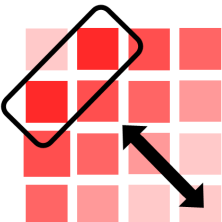

(d)

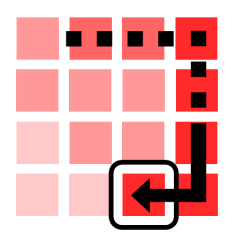

(e)

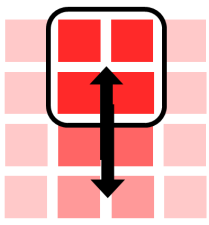

(f)

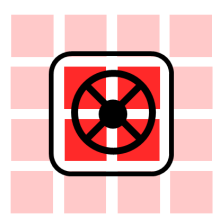

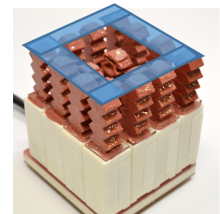
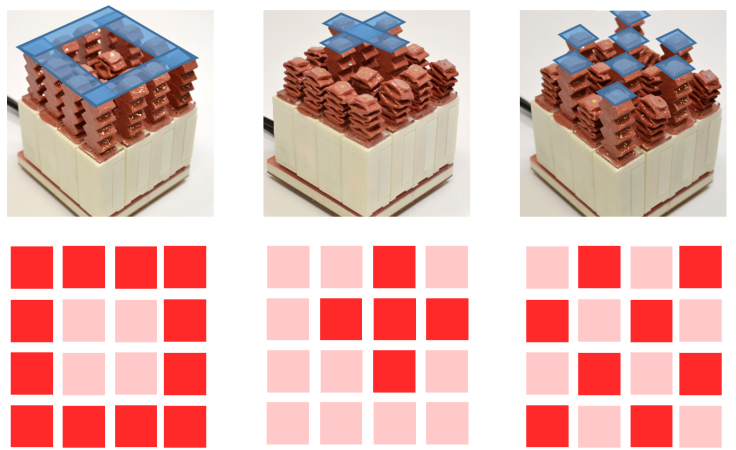

Cross

Checkered

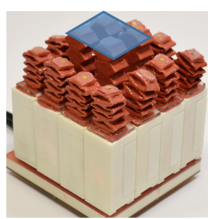

Box
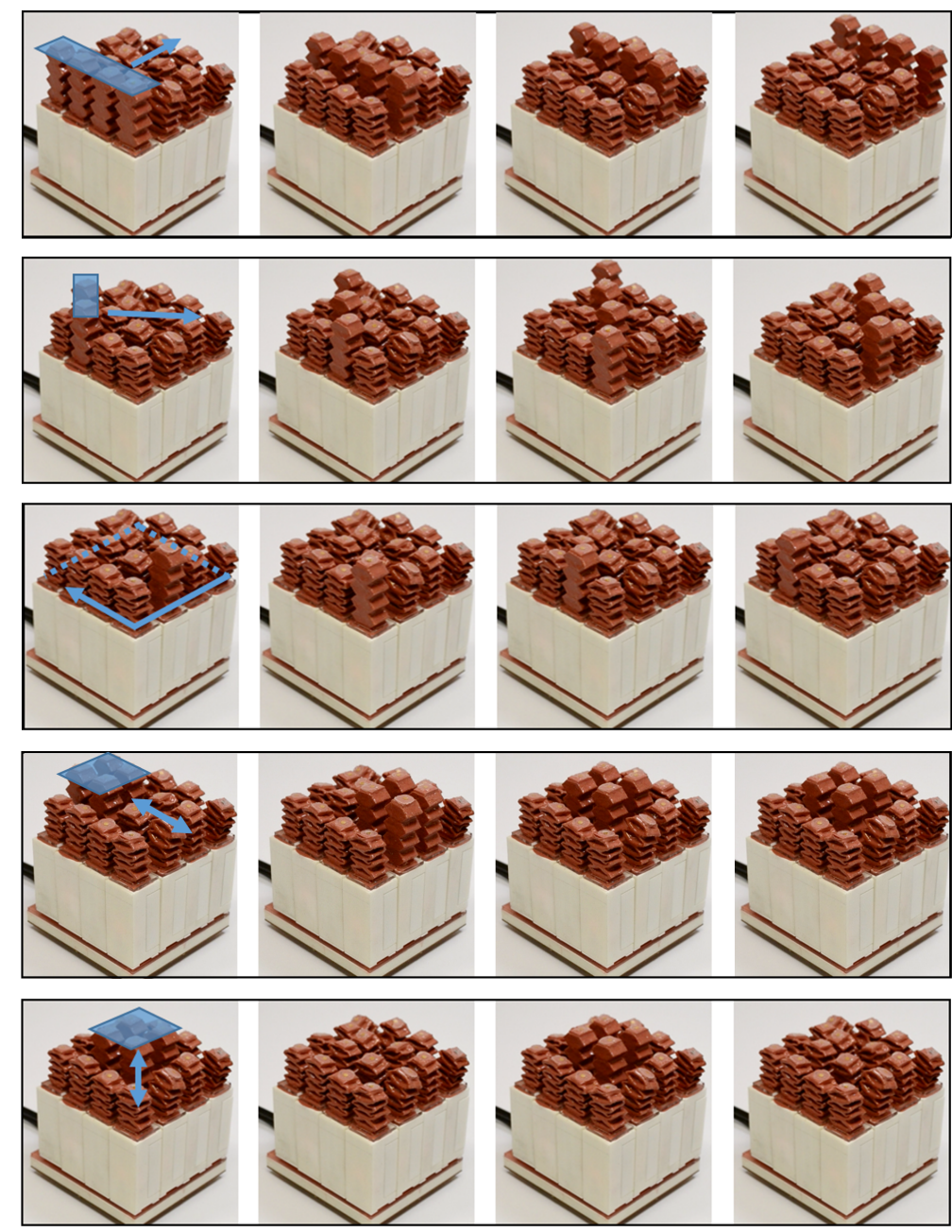

Time

Fig. 6. The SRS can render a variety of shapes and patterns which can be discerned by visual display or through safe tactile interaction to convey information or feedback to a user. A selection of static shapes are depicted in (a), and examples of dynamic patterns are shown including, (b) orthogonal wave, (c) diagonal wave, (d) path trace, (e) moving shape, and (f) blinking shape, although many more alternatives are possible with the high-DoF reconfigurable surface.

static shape reconfiguration or dynamic pattern propagation. As the active surface of the device consists of an inherently soft structure, it can be safely operated in direct or close contact with sensitive body areas where the structural data representations may be perceived. Using shapes, patterns, and motion profiles, a variety of information could be transmitted to a user. This method of haptic representation could be used to render geometric or non-geometrical data. This includes topological graphics, simulated structures, feedback from other devices, or incoming communication.

We implemented some examples to illustrate the possible application toward a tactile display device. Fig. 6 shows both static and dynamically rendered shapes and patterns. These were generated with the prototype to form at least ten unique 
(a)

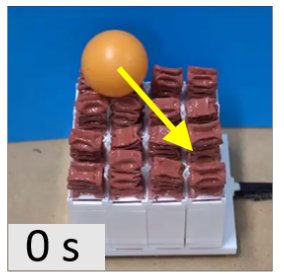

(b)

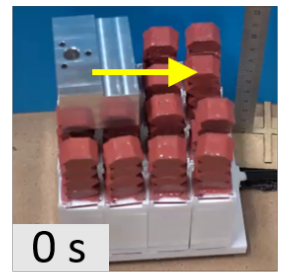

(c)

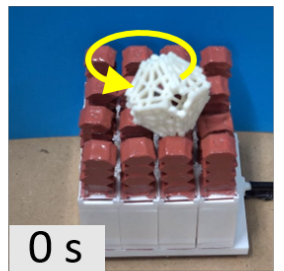

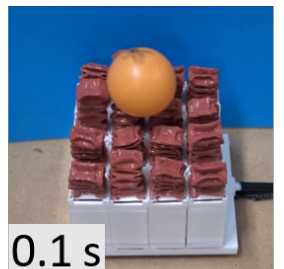
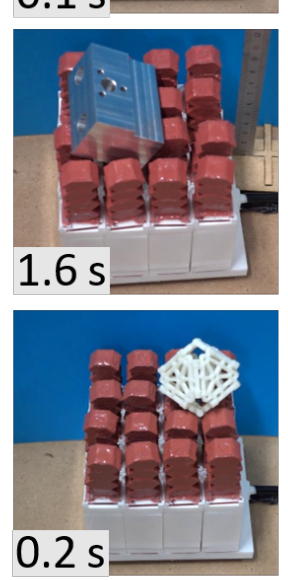
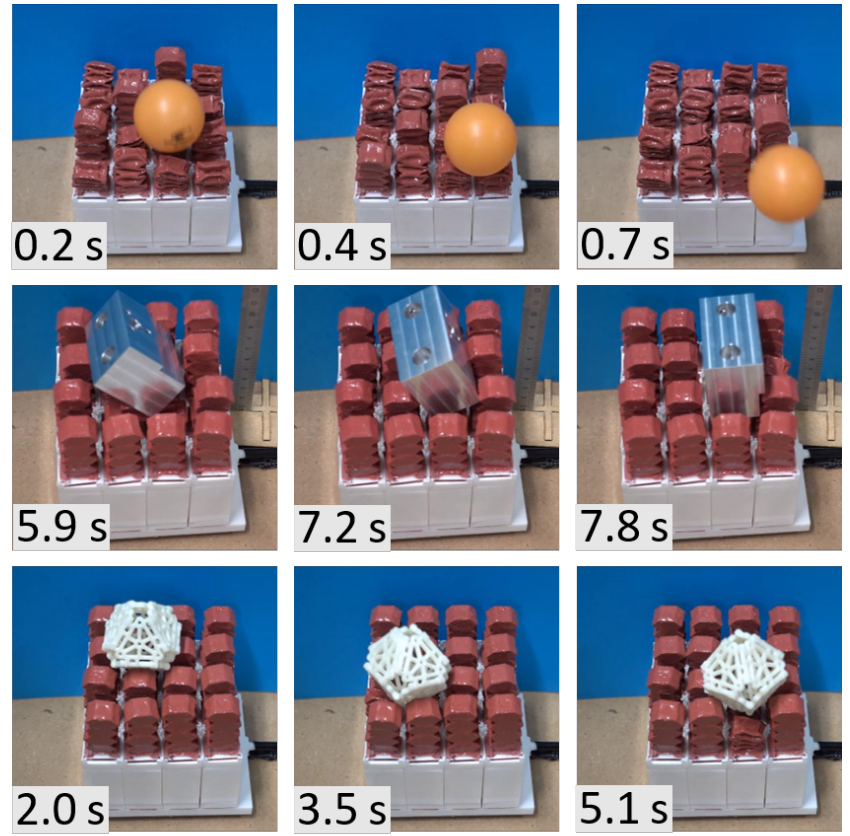

Fig. 7. Three objects were transported across the SRS with different dynamic shape patterns: a lightweight ball transported via positive (convex) diagonal wave (a), a heavy block via positive orthogonal wave (b), and a delicate object transported in a circular path with negative (concave) wave along a traced path (c).

tactile primitives which could be used to encode information. Using these primitives in combinations to form tactile words, an even wider range of complex tactile communication is possible.

2) Object Manipulation: Surface shape patterns can also convey and manipulate physical objects. By producing variable height profiles, the SRS is capable of directing the motion of objects placed on it from high to low spots, leveraging gravity. As the topology of the surface changes dynamically, the position and orientation of any objects resting on it follows the low-energy wells formed around prominent features, allowing the objects to be moved either by rolling or sliding in the direction of traveling waves. In addition, static features may be produced to provide passive object constraints that can be used to guide objects in motion or arrest them.

We demonstrate these capabilities experimentally using the SRS prototype to manipulate three different types of objects categorized as lightweight, heavy, and delicate, respectively, as shown in Fig. 7. Both negative and positive waves were tested to transport the objects; the former produced by the progression of a concave feature across otherwise nominally extended actuators, with the latter formed by a convex feature traveling across a surface of otherwise fully retracted actuators. Both methods were effective in transporting objects across the surface, although a trade-off was found between speed and controllability for certain objects which would prescribe the advantage of one over the other depending on application requirements. This difference is mainly due to the presence or lack of fully defined object support by the surface during object transport. With negative waves, objects maintain quasistatic equilibrium throughout the process of translation, being borne by multiple extended actuators surrounding a concave surface feature, allowing better control. For positive traveling waves, a prominent convex feature is used instead to push an object across the surface, leading to behavior closer to continuous falling for less controllable but more dynamic and high speed transport. This was particularly apparent through observations of the movement of a lightweight spherical object which is prone to rolling freely away from a prominent, positive wave at high speed, while a negative wave maintained control of the object at all times. The average linear velocity of the ball-shaped object in translation was estimated to be 27.5 $\mathrm{cm} / \mathrm{s}$, while the large, heavy, rectangular object was observed to be $0.7 \mathrm{~cm} / \mathrm{s}$.

\section{Stiffness Mode: tunable passive compliance}

The variable stiffness behavior of the actuators as measured in Section II-B can also be leveraged in combination with shape and pattern rendering to augment the position control capabilities mode described above. The nominal stiffness of the surface can be tuned for either application by adjusting the nominal range of operation for the actuators. At different overall displacement levels or actuator heights, the surface can render the same features used for tactile display or object manipulation within controllable regimes of compliance. This feature extends the utility of both by adding additional haptic information or mechanical control. While integrated sensing can also be used to achieve active force control for the same purpose, the method of tuning the passive stiffness of an SRS allows a more robust guarantee of safety for objects in contact, independent of control system dynamics and speed which affect other active stiffness control methods. 


\section{DISCUSSION}

In this paper we present a new reconfigurable surface concept based on a new type of modular, linear vacuumpowered soft pneumatic actuator (linear V-SPA), which is capable of operating in position, force and stiffness control modes. A 16-DoF prototype was experimentally tested for validation of the control modes in the context of realistic potential applications. The design is fundamentally modular and extensible to different footprints with high numbers of modules.

Using force sensors on the surface of each actuator, closedloop force control was utilized to actively modulate the interface pressure between the SRS and non-uniform objects in contact. This mode was was able to reduce and redistribute the forces on the surface. This could enable applications such as mechanical human interface devices to alleviate discomfort or tissue damage from extended duration pressure concentration from wearable robots or from body weight support.

In open-loop position control mode, the SRS also demonstrated the ability to render physical shapes and patterns to function as a non-visual tactile display. Static and dynamic primitives were generated which could serve as elements for a tactile language. These could convey complex information for use in machine feedback or communication applications. While not demonstrated here, the force control mode could also be combined with this application to provide another level of feedback, or the sensors could be used for touch driven device input. The position control also enabled the transport of physical objects. By creating traveling waves along the surface, the SRS is able to manipulate objects through rolling. This could enable industrial conveyor applications for delicate objects. We also demonstrated the functionality of the SRS as a variable stiffness device. This control mode could be combined with position control to create haptic displays with controllable passive stiffness. It could also be used to passively mitigate impacts or dampen motion without the need or limitations of active stiffness control.

As a result of a modular architecture and the use of soft material actuators powered by vacuum to provide safe and compliant reconfigurability, the SRS presented here demonstrates a unique combination of performance and design efficiency. With a diverse range of potential functions along with a compact and scalable design, the multi-functional SRS device enables new and extended applications limited in previous similar devices by practical size or actuator performance constraints. The unique features of the SRS afford benefits to high-DoF manipulation, interaction, or communication tasks with potential in both industrial and personal use by providing safe and compliant reconfigurability.

\section{ACKNOWLEDGMENT}

This work was supported by the Swiss National Science Foundation (SNSF), the EU FLAG-ERA project RoboCom++ and by the National Centre of Competence in Research (NCCR) Robotics (Switzerland).

\section{REFERENCES}

[1] D. J. Page, "Reconfigurable surface," U.S. Patent US6903871 B2, Jun., 2005, u.S. Classification 359/443, 359/446, 359/451, 359/277, 359/893; International Classification B29C45/00, B29C33/30; Cooperative Classification B29C45/00, G03B21/56, B29C33/308, B29C33/307; European Classification B29C33/30F. [Online]. Available: http://www.google.com/patents/US6903871

[2] B. Peters and E. Marion, "Methods and apparatus for a reconfigurable surface," U.S. Patent US20 120279812 A1, Nov., 2012, u.S. Classification 188/67, 700/275; International Classification F16D63/00, F16D59/00, G05B15/02; Cooperative Classification G05B19/4099, G05B2219/49025, B21D37/02, F16D63/008. [Online]. Available: http://www.google.com/patents/US20120279812

[3] M. K. Rasmussen, E. W. Pedersen, M. G. Petersen, and K. Hornb $\backslash$ a ek, "Shape-changing Interfaces: A Review of the Design Space and Open Research Questions," in Proceedings of the SIGCHI Conference on Human Factors in Computing Systems, ser. CHI '12. New York, NY, USA: ACM, 2012, pp. 735-744. [Online]. Available: http://doi.acm.org/10.1145/2207676.2207781

[4] S. Follmer, D. Leithinger, A. Olwal, A. Hogge, and H. Ishii, "inFORM: dynamic physical affordances and constraints through shape and object actuation." in Uist, vol. 13, 2013, pp. 417-426.

[5] I. Poupyrev, T. Nashida, S. Maruyama, J. Rekimoto, and Y. Yamaji, "Lumen: Interactive Visual and Shape Display for Calm Computing," in ACM SIGGRAPH 2004 Emerging Technologies, ser. SIGGRAPH '04. New York, NY, USA: ACM, 2004, pp. 17-. [Online]. Available: http://doi.acm.org/10.1145/1186155.1186173

[6] D. Leithinger and H. Ishii, "Relief: A Scalable Actuated Shape Display," in Proceedings of the Fourth International Conference on Tangible, Embedded, and Embodied Interaction, ser. TEI '10. New York, NY, USA: ACM, 2010, pp. 221-222. [Online]. Available: http://doi.acm.org/10.1145/1709886.1709928

[7] H. Iwata, H. Yano, F. Nakaizumi, and R. Kawamura, "Project FEELEX: Adding Haptic Surface to Graphics," in Proceedings of the 28th Annual Conference on Computer Graphics and Interactive Techniques, ser. SIGGRAPH '01. New York, NY, USA: ACM, 2001, pp. 469-476. [Online]. Available: http://doi.acm.org/10.1145/383259.383314

[8] G. M. Troiano, J. Tiab, and Y.-K. Lim, "SCI-FI: Shape-Changing Interfaces, Future Interactions," in Proceedings of the 9th Nordic Conference on Human-Computer Interaction, ser. NordiCHI '16. New York, NY, USA: ACM, 2016, pp. 45:1-45:10. [Online]. Available: http://doi.acm.org/10.1145/2971485.2971489

[9] D. Leonardis, L. Claudio, and A. Frisoli, "A survey on innovative refreshable braille display technologies," in AHFE International Conference on Applied Human Factors and Ergonomics, Advances in Design for Inclusion, 2017.

[10] L. Yobas, D. M. Durand, G. G. Skebe, F. J. Lisy, and M. A. Huff, "A novel integrable microvalve for refreshable Braille display system," Journal of Microelectromechanical Systems, vol. 12, no. 3, pp. 252-263, Jun. 2003.

[11] T. S. Prince, G. G. Skebe, F. J. Lisy, and R. N. Schmidt, "Refreshable braille display system with a flexible surface," U.S. Patent US6743021 B2, Jun., 2004, u.S. Classification 434/113, 434/112; International Classification G09B21/00; Cooperative Classification G09B21/003; European Classification G09B21/00B3. [Online]. Available: http://www.google.com/patents/US6743021

[12] A. Russomanno, R. B. Gillespie, S. O’Modhrain, and M. Burns, "The design of pressure-controlled valves for a refreshable tactile display," in World Haptics Conference (WHC), 2015 IEEE. IEEE, 2015, pp. 177-182.

[13] S. Lee, K. Jung, J. Koo, S. Lee, H. Choi, J. Jeon, J. Nam, and H. Choi, "Braille display device using soft actuator," in Smart Structures and Materials 2004: Electroactive Polymer Actuators and Devices (EAPAD), vol. 5385. International Society for Optics and Photonics, 2004, pp. 368-380.

[14] P. Yang, "Electroactive polymer actuator braille cell and braille display," Apr. 19 2005, uS Patent 6,881,063.

[15] K. Ren, S. Liu, M. Lin, Y. Wang, and Q. M. Zhang, "A compact electroactive polymer actuator suitable for refreshable Braille display," Sensors and Actuators A: Physical, vol. 143, no. 2, pp. 335-342, May 2008. [Online]. Available: http://www.sciencedirect.com/science/article/pii/S0924424707008114

[16] Y. Haga, W. Makishi, K. Iwami, K. Totsu, K. Nakamura, and M. Esashi, "Dynamic braille display using sma coil actuator and magnetic latch," Sensors and Actuators A: Physical, vol. 119, no. 2, pp. 316-322, 2005. 
[17] D. Chaves, I. Peixoto, A. Lima, M. Vieira, and C. De Araujo, "Microtuators of sma for braille display system," in Medical Measurements and Applications, 2009. MeMeA 2009. IEEE International Workshop on. IEEE, 2009, pp. 64-68.

[18] P. Sapra, A. K. Parsurampuria, D. Gupta, S. Muralikrishnan, M. Raj, A. Anand, V. Darda, R. Paul, M. Balakrishnan, and P. Rao, "A compliant mechanism design for refreshable braille display using shape memory alloy," in ASME 2015 International Design Engineering Technical Conferences and Computers and Information in Engineering Conference. American Society of Mechanical Engineers, 2015, pp. V009T07A054V009T07A054.

[19] A. A. Stanley and A. M. Okamura, "Controllable surface haptics via particle jamming and pneumatics," IEEE Transactions on Haptics, vol. 8, no. 1, pp. 20-30, 2015.

[20] A. A. Stanley, J. C. Gwilliam, and A. M. Okamura, "Haptic jamming: A deformable geometry, variable stiffness tactile display using pneumatics and particle jamming," in 2013 World Haptics Conference (WHC), Apr. 2013, pp. 25-30.

[21] W. Nutzel, "Display surface for tactile information," U.S. Patent US5 086287 A, Feb., 1992, u.S. Classification 340/407.1, 434/112, 434/115, 434/113; International Classification G09B21/00; Cooperative Classification G09B21/003; European Classification G09B21/00B3. [Online]. Available: http://www.google.com/patents/US5086287

[22] I. M. Koo, K. Jung, J. C. Koo, J.-D. Nam, Y. K. Lee, and H. R. Choi, "Development of soft-actuator-based wearable tactile display," IEEE Transactions on Robotics, vol. 24, no. 3, pp. 549-558, 2008.

[23] H. A. Sonar and J. Paik, "Soft pneumatic actuator skin with piezoelectric sensors for vibrotactile feedback," Frontiers in Robotics and AI, vol. 2, p. $38,2016$.

[24] M. Raitor, J. M. Walker, A. M. Okamura, and H. Culbertson, "Wrap: Wearable, restricted-aperture pneumatics for haptic guidance," in Robotics and Automation (ICRA), 2017 IEEE International Conference on. IEEE, 2017, pp. 427-432.

[25] R. M, G. SS, and R. PA, "Preventing pressure ulcers: A systematic review," JAMA, vol. 296, no. 8, pp. 974-984, 2006. [Online]. Available: + http://dx.doi.org/10.1001/jama.296.8.974

[26] G. Votolato, Transport design: a travel history, 2007.

[27] "WaveHandling | Festo Corporate," 2014. [Online]. Available: https://www.festo.com/group/en/cms/10225.htm

[28] B. Mosadegh, A. D. Mazzeo, R. F. Shepherd, S. A. Morin, U. Gupta, I. Zhalehdoust Sani, D. Lai, S. Takayama, and G. M. Whitesides, "Control of soft machines using actuators operated by a Braille display," Lab on a Chip, vol. 14, no. 1, pp. 189-199, 2014. [Online]. Available: http://pubs.rsc.org/en/Content/ArticleLanding/2014/LC/C3LC51083B

[29] Z. Deng, M. Stommel, and W. Xu, "A Novel Soft Machine Table for Manipulation of Delicate Objects Inspired by Caterpillar Locomotion," IEEE/ASME Transactions on Mechatronics, vol. 21, no. 3, pp. 17021710, Jun. 2016.

[30] D. F. Walczyk and D. E. Hardt, "Design and analysis of reconfigurable discrete dies for sheet metal forming," Journal of Manufacturing Systems, vol. 17, no. 6, pp. 436-454, Jan. 1998. [Online]. Available: http://www.sciencedirect.com/science/article/pii/S027861259980003X

[31] B. J. Peters, "Design and fabrication of a digitally reconfigurable surface," Thesis, Massachusetts Institute of Technology, 2011. [Online]. Available: http://dspace.mit.edu/handle/1721.1/68541

[32] P. L. Hoffman, "Method for forming composite parts using reconfigurable modular tooling," Dec. 8 1998, uS Patent 5,846,464.

[33] "Applications of the reconfigurable mould, the adaptive mould." [Online]. Available: http://adapa.dk/applications/

[34] Z. Szabo and E. T. Enikov, "Development of Wearable Micro-actuator Array for 3-D Virtual Tactile Displays," Journal of Electromagnetic Analysis and Applications, vol. 04, p. 219, Jun. 2012.

[35] M. A. Robertson and J. Paik, "New soft robots really suck: Vacuum-powered systems empower diverse capabilities," Science Robotics, vol. 2, no. 9, 2017. [Online]. Available: http://robotics.sciencemag.org/content/2/9/eaan6357

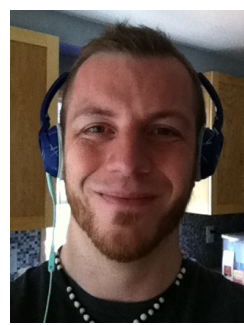

Matthew A. Robertson is a $\mathrm{PhD}$ candidate in the Reconfigurable Robotics Laboratory (RRL) at EPFL, currently active in the fields of Soft Robotics and Wearable Devices. His research involves the design, development, and characterization of new softmaterial-based actuators, along with their unique application in versatile robotic systems. Beyond this current focus, he is also generally interested in dynamic robotic legged locomotion, and hueristic design and control strategies. Prior to entering his $\mathrm{PhD}$ program, he worked as a research engineer with a startup company designing, fabricating, and testing advanced robotic prosthetic devices. Matthew received a Master of Science in Mechanical Engineering degree from the University of Michigan (UM), Ann Arbor and a Bachelor of Science degree from the Massachusetts Institute of Technology (MIT).

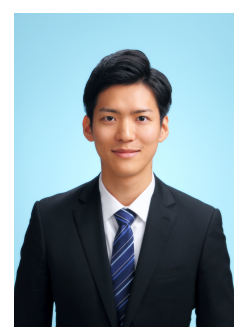

Masato Murakami received his Masters degree in Mechanical Engineering from the University of Tokyo Creative Design Lab. (Nakao Nagato Ueda Osa Lab.). He currently works for the Japanese car company Toyota as an engineer in the field of fuel cell manufacturing technology. His research experience focuses on manufacturing processes, especially for aluminum die-casting and fuel cells.

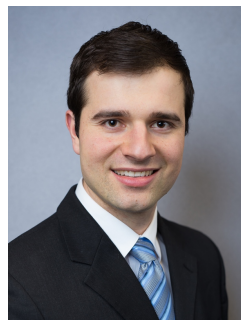

Wyatt Felt is a Postdoctoral Fellow in the Reconfigurable Robotics Laboratory (RRL) at EPFL. $\mathrm{He}$ received a $\mathrm{PhD}$ in Mechanical Engineering (Aug. 2017) from the University of Michigan where he was a NSF Graduate Research Fellow. Prior to his $\mathrm{PhD}$, he worked as Head of Product Development at Owlet Baby Monitors (owletcare.com). He received a B.S. in Mechanical Engineering in 2013 from Brigham Young University (BYU)

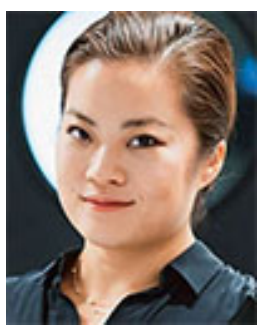

Jamie Paik (M07) received the Ph.D. degree in designing a humanoid arm and hand from the Seoul National University, Seoul, South Korea, in 2007. She is the Director and the Founder of the Reconfigurable Robotics Lab (RRL), Ecole Polytechnique $F$ ed erale de Lausanne, Lausanne, Switzerland, and a core member of the Swiss National Centre of Competence in Research (NCCR) in Robotics. During her Postdoctoral po- sitions at the Institut des Systems Intelligents et de Robotic, Universitat Pierre Marie Curie, Paris VI, France, she devel- oped laparoscopic tools that are internationally patented and commercialized. At the Microrobotics Laboratory, Harvard University, Cambridge, MA, USA, she started developing unconventional robots that push the physical limits of materials and mechanisms. Her latest research efforts are in soft robotics and self-morphing Robogami (robotic orgami) that transforms planar shapes to 3-D by folding in predefined patterns and sequences, just like the paper art, origami. 